\title{
Strongly Coupled Plasmas in High-Energy Physics
}

\author{
Markus H.Thoma \\ Centre for Interdisciplinary Plasma Science \\ Max-Planck-Institut für extraterrestrische Physik \\ P.O. Box 1312, D-85741 Garching, Germany \\ Email: thoma@mpe.mpg.de
}

\begin{abstract}
One of the main activities in high-energy and nuclear physics is the search for the so-called quark-gluon plasma, a new state of matter which should have existed a few microseconds after the Big Bang. A quark-gluon plasma consists of free color charges, i.e. quarks and gluons, interacting by the strong (instead of electromagnetic) force. Theoretical considerations predict that the critical temperature for the phase transition from nuclear matter to a quark-gluon plasma is about 150 - $200 \mathrm{MeV}$. In the laboratory such a temperature can be reached in a so-called relativistic heavy-ion collision in accelerator experiments. Using the color charge instead of the electric charge, the Coulomb coupling parameter of such a system is of the order $10-30$. Hence the quark-gluon plasma is a strongly coupled, relativistic plasma, in which also quantum effects are important. In the present work the experimental and theoretical status of the quark-gluon plasma physics will be reviewed, emphasizing the similarities and differences with usual plasma physics. Furthermore, the mixed phase consisting of free quarks and gluons together with hadrons (e.g. pions) will be discussed, which can be regarded as a complex plasma due to the finite extent of the hadrons.
\end{abstract}

\section{BASIC FACTS OF QUARK, GLUONS, AND QUANTUM CHROMODYNAMICS}

One of the main tasks of modern nuclear and high-energy physics is the quest of the quark-gluon plasma (QGP), a new state of matter, predicted to exist at high temperatures and densities. Quarks have been proposed in the middle of the sixties as elementary substructure particles of the strongly interacting particles, the so called hadrons, in order to explain the large spectrum of hadrons discovered at this time [1]. Quarks are fermions, carrying the spin $1 / 2$. Nowadays, 6 different quark species, characterized by the quantum number flavor (up, down, strange, charm, bottom, top) are known. These quark flavors cover a wide regime of masses, from about $5 \mathrm{MeV}$ for the up-quark to $170 \mathrm{GeV}$ for the top-quark. In addition, quarks have an electric charge, either $2 / 3$ or $-1 / 3$ of the electron charge.

There are two different combinations of quarks for building hadrons: baryons, i.e. hadrons which are fermions, e.g. protons and neutrons, are fomed by 3 quarks, whereas mesons, which are bosons, e.g. pions, consist of a quark and an anti-quark. Atomic nuclei contain only protons and neutrons, which are build up of up- and down-quarks. The other (heavier) quark flavors exist only in short-living hadrons, created in accelerator experiments or cosmic rays. So far no free quarks have been observed in nature, a phenomenon called confinement.

For explaining the hadron spectrum as well as cross sections for the hadron production in electron-positron annihilations, additional quark states had to be introduced. These states are characterized by the quantum number color, which exists in three different possibilities (red, blue, green). In the middle of the seventies, it was realized, that this quantum number can be regarded as the charge of the strong interaction between the quarks. This led to the formulation of a theory for the quark interaction, called Quantum Chromodynamics (QCD) [2], analogously to QED, describing the electromagnetic interaction. For example, in QED the scattering of two electrons is mediated by the exchange of a photon. The exchange particles of QCD, mediating the strong interaction, are called gluons. The main difference between QED and QCD is the fact that quarks come in three different colors, whereas electrons carry only one charge. Mathematically this implies that QED is based on the abelian $U(1)$ gauge group, while QCD on the non-abelian $S U(3)$. As a consequence, gluons carry a color charge, in contrast to photons which have no electric charge. Therefore, gluons can interact directly with other gluons. A consequence of this gluon self-interaction is the fact that, in contrast to QED, the interaction strength of two color charges increases with the distance between these partons. (A parton is either a quark or gluon.) Then, for instance, a meson can be pictured as a rubber band with a quark on one end and an anti-quark on the other. If the quarks are close together, they do not feel each other. This phenomenon is called asymptotic freedom, which can be derived directly from QCD. If the distance between the quark and the anti-quark gets larger, however, the force between them increases, until the rubber band breaks into two new mesons, e.g. in highenergy scattering experiments. In this way, one can explain confinement: the interaction between hadrons can lead only to new hadrons but not to freely propagating quarks. In other words, only colorless states, the hadrons, can exist as free particles. However, it should be noted that confinement has not been proved rigorously from first principles.

Following this picture, hadrons can be considered as quark bags in which the quarks are confined by their interaction with each other. Since the interaction is mediated by gluons which also can split into virtual quark anti-quark pairs, the hadrons are complicated systems containing quarks and gluons (see Fig.1). A nucleus is now represented by a dense system of individual quark bags, the protons and neutrons. 


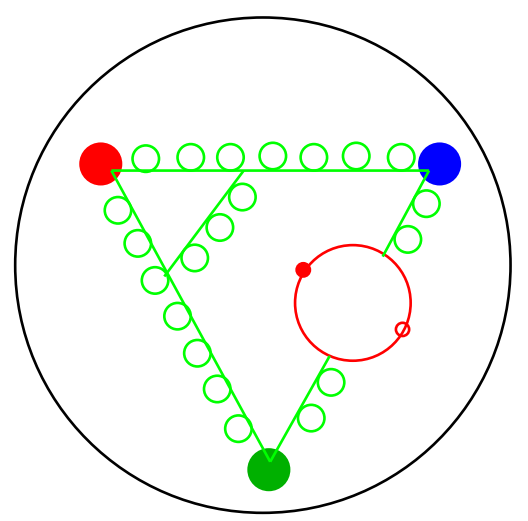

Fig. 1. A baryon as quark bag containing three quarks interacting via gluon exchange

\section{QUARK-GLuON Plasma: EXPERIMENT AND THEORY}

Shortly after quarks were proposed the question arose, what will happen if we compress the nucleus, i.e. increase the nuclear or baryon density, or if we heat up the nucleus in a fixed volume to such high temperatures that pair creation (e.g. of pions) occurs in scattering events of hadrons with large thermal energies. Then the system becomes denser and the bags should start to overlap and to dissolve, transforming the nucleus into a "soup" of freely propagating quarks and gluons as sketched in Fig.2, which was called a quark-gluon plasma [3]. Theoretical estimates predicted a critical baryon density of about 10 times normal nuclear matter density, $\rho_{0}=0.125$ $\mathrm{Gev} / \mathrm{fm}^{3}$ corresponding to $2.2 \times 10^{17} \mathrm{~kg} / \mathrm{m}^{3}$. The critical temperature $T_{c}$ of this deconfinement phase transition from hadronic matter to the QGP phase should be of the order 150 to $200 \mathrm{MeV}\left(1.8\right.$ to $\left.2.4 \times 10^{12} \mathrm{~K}\right)$.

The phase diagram of nuclear matter is pictured in Fig.3, where the phase transition line in the plane given by the temperature and baryon density is shown. Also three different possibilities to cross this border line are indicated. Until about a few $\mu$ s after the Big Bang the Early Universe had a temperature above the critical temperature and should have existed in the QGP phase. In the center of neutron stars, on the other hand, the baryon density might exceed the critical one at low temperatures. Then the interior of these compact stars might actually consist of degenerate quark matter instead of neutron matter ${ }^{1}$. Finally in high-energy nucleus-nucleus collisions, also called relativistic heavy-ion collisions, a dense and hot fireball is created for a short time, which might be also in the QGP phase [4].

Different accelerator experiments have been and will be performed for searching for the QGP. For producing a large and hot fireball, heavy-ion collisions with $\mathrm{Au}-, \mathrm{Pb}-$, and Ubeams at center of mass energies from 4 to $5500 \mathrm{GeV}$ per nucleon have been or will be conducted at the Brookhaven

\footnotetext{
${ }^{1}$ As a matter of fact, recent investigations revealed that there might be a quark pairing (color superconductivity) at high quark densities, which could have an influence on the properties of neutron stars containing quark matter (see e.g. Ref.[5]).
}

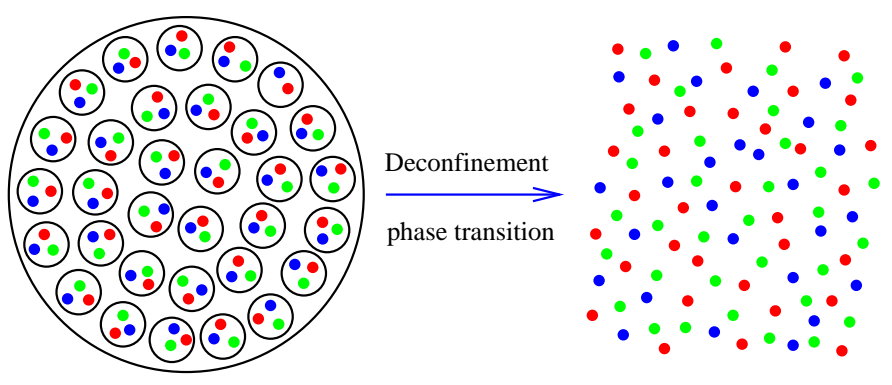

Fig. 2. The deconfinement phase transition from nuclear matter to QGP

National Laboratory on Long Island (AGS, RHIC) and at CERN in Switzerland (SPS, LHC). At the accelerator SPS first indications for the discovery of the QGP plasma have been found and announced at CERN in 2000 [6]. Also in 2000 the dedicated heavy-ion experiment RHIC started its operation with energies up to $200 \mathrm{GeV}$ per nucleon, which is about 10 times the energy of SPS. Due to the huge amount of data - thousands of particle tracks are produced in a single collision - the analysis of the data is still in progress, although preliminary results confirm the conclusions from SPS. The high density phase at moderate temperatures will be addressed at a future accelerator facility at the GSI Darmstadt, which eventually may allow to study the superconducting phase of QCD.

Theoretical estimates for these collisions predicted a maximum temperature clearly above the critical at RHIC $\left(T_{\max }>\right.$ $230 \mathrm{MeV}$ ), a maximum volume of the hot fireball of about $3000 \mathrm{fm}^{3}$, containing about 10000 partons, and a life-time of the QGP of about 5-10 fm/c $\left(1 \mathrm{fm} / \mathrm{c}\right.$ corresponds to $3 \times 10^{-24}$ s). This short life-time is caused by the fast expansion of the fireball leading to a fast reduction of the maximum temperature and the phase transition to the hadronic state. The pre-equilibrium phase, i.e., the time needed to form an equilibrated fireball after the collision of the cold nuclei, was estimated to be of the order of only $1 \mathrm{fm} / \mathrm{c}$ due to the strong interaction between the particles. Hence, the chances to create an equilibrated QGP in relativistic heavy-ion collisions seem to be good.

However, the main problem lies in the detection of this state since the QGP, living only for an extremely short period in a small spatial volume, cannot be observed directly. Only by comparing theoretical predictions for signatures of the QGP, such as the spectra of the emitted particles (photons, leptons, and hadrons) which reach the detector after the decay of the expanding fireball, with experimental data, the QGP can be discovered. Unfortunately, so far no unambiguous signature has been proposed due to the complexity of the strongly interacting many-body system. Therefore, the final identification of the QGP might not be possible by a unique signature but rather by circumstantial evidence owing to a combination of all possible signatures.

The theoretical description of the QGP has to take into account that it is a strongly coupled system at temperatures reachable in heavy-ion collisions. At $T \simeq 200 \mathrm{MeV}$ the strong 


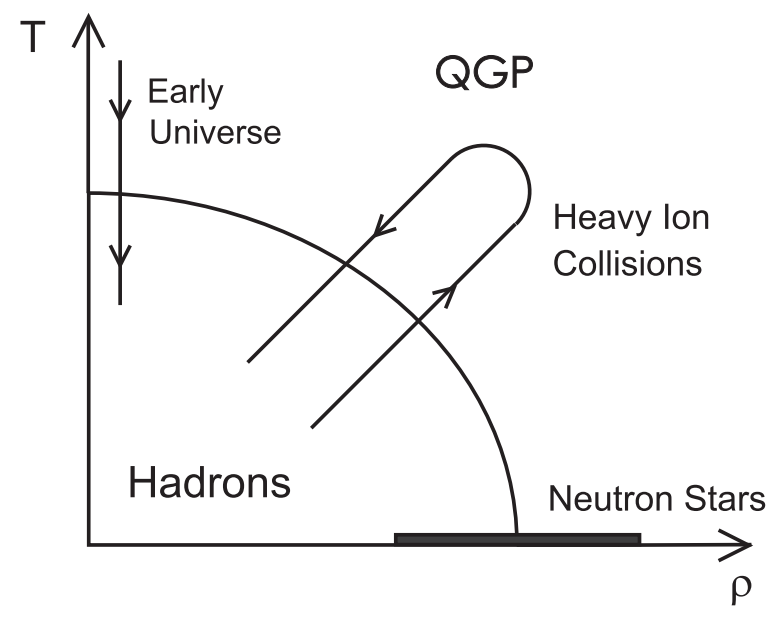

Fig. 3. Phase diagram of nuclear matter (temperature vs. baryon density)

coupling constant $g$, corresponding to the electromagnetic coupling $e$, assumes values of $g \simeq 1.5-2.5$ in natural units ( $\hbar=c=k_{B}=1$ ). The strong coupling constant is temperature dependent and decreases with increasing temperature and density because of asymptotic freedom, i.e. the QGP becomes an ideal plasma in the infinitely high temperature limit. At $T \simeq 200 \mathrm{MeV}$, however, the QGP is a non-ideal plasma. Its Coulomb coupling parameter is given by the chromoelectrostatic interaction energy divided by the thermal parton energy ${ }^{2}$

$$
\Gamma=\frac{C g^{2}}{\Delta T},
$$

where $\Delta$ is the distance between the partons, following approximately from the number density $n \sim T^{3}$ assuming the Stefan-Boltzmann limit (ideal gas of massless particles). At $T=200 \mathrm{MeV}$ the distance is of the order $\Delta=0.5$ fm. The constant $C=3$ for gluons and $4 / 3$ for quarks come from the different color charges of gluons and quarks, respectively. Typical values of $\Gamma$ for temperatures, which can be achieved in heavy-ion collisions, are between 10 and 30, corresponding to a strongly coupled plasma. For $T \rightarrow \infty$ the coupling parameter approaches zero, although $\Delta \sim 1 / T$ since $g(T=\infty)=0$, which demonstrates the ideal plasma limit at high temperatures.

Furthermore, the QGP is a relativistic system as the masses of its components are much smaller than their thermal energies which are of the order of the temperature. Gluons are massless, while the up- and down-quarks have masses of a few MeV. Only the mass of the strange-quark $\left(m_{s} \simeq 150 \mathrm{MeV}\right)$ is comparable to the temperature. The other quark flavors have much higher masses and are therefore not thermally exited, i.e., they are suppressed compared to up-, down, and strangequarks in the QGP.

Finally, at temperatures not too far from the critical, quantum effects play an important role in the QGP. Hence a

\footnotetext{
${ }^{2}$ Of course, in the relativistic case, the (chromo)magnetic interaction becomes equally important.
}

theoretical description has to start from a statistical quantum field theory, i.e. thermal QCD. Due to the large coupling constant non-perturbative methods are required. The only well-established non-perturbative approach for quantum field theories are lattice simulations, where the quantum theoretical equations of motions are solved numerically on a 4 dimensional space-time grid using Monte-Carlo techniques [7]. This method has the advantage that it works for all temperatures below as well as above the phase transition, allowing, for example, to determine the critical temperature, the order of the phase transition, or the equation of state of the QGP. However, it is very difficult to compute dynamical quantities such as most of the signatures of the QGP which are based on particle production rates or similar dynamical processes. Hence, perturbative methods, extended to finite temperatures and improved by resummation techniques, are also adopted to estimate dynamical properties and signatures of the QGP [8]. Although they might be valid only at temperatures far above the critical one, these methods give at least important insights in the dynamical structure of the QGP.

In the high temperature limit $\left(T \gg T_{c}\right.$ corresponding to $g \ll 1)$, perturbation theory can be shown to be equivalent to the semiclassical Vlasov equation [9], generalized to the relativistic case and the strong instead of electromagnetic interaction. The only quantum input comes from using the Bose distribution for gluons and the Fermi distribution for quarks instead of the Maxwell distribution. As in usual plasma physics one can derive, for instance, the dielectric tensor from the Vlasov equation. In the case of an isotropic and homogeneous system this tensor has only two independent components (longitudinal and transverse dielectric functions), which depend on the energy (frequency) $\omega$ and momentum (wave number) $k$ :

$$
\begin{aligned}
& \epsilon_{L}(\omega, k)=1+\frac{3 m_{g}^{2}}{k^{2}}\left(1-\frac{\omega}{2 k} \ln \frac{\omega+k}{\omega-k}\right) \\
& \epsilon_{T}(\omega, k)=1-\frac{3 m_{g}^{2}}{k^{2}}\left[1-\left(1-\frac{k^{2}}{\omega^{2}}\right) \frac{\omega}{2 k} \ln \frac{\omega+k}{\omega-k}\right],
\end{aligned}
$$

where $m_{g}^{2}=g^{2} T^{2} / 3\left(1+N_{f} / 6\right)$ is called the effective gluon mass with $N_{f}$ thermally excited quark flavors in the plasma. The same result can be obtained from the gluon self-energy in lowest order perturbation theory in the high temperature limit. Going beyond this approximation, perturbative QCD allows a systematic treatment of higher order effects, such as damping rates coming from collisions between the particles. However, in order to avoid infrared singularities due to the exchange of massless gluons and violations of the gauge invariance, resummation techniques have to be adopted at finite temperature [8]. The development of such methods is a very active research topic.

From the chromoelectromagnetic Maxwell equations one obtains the constraints on the dielectric functions, $\epsilon_{L}(\omega, k)=$ 0 and $\epsilon_{T}(\omega, k)=k^{2} / \omega^{2}$, which describe the propagation of collective plasma modes. From these equations dispersion relations $\omega_{L, T}(k)$ for longitudinal and transverse plasma modes 


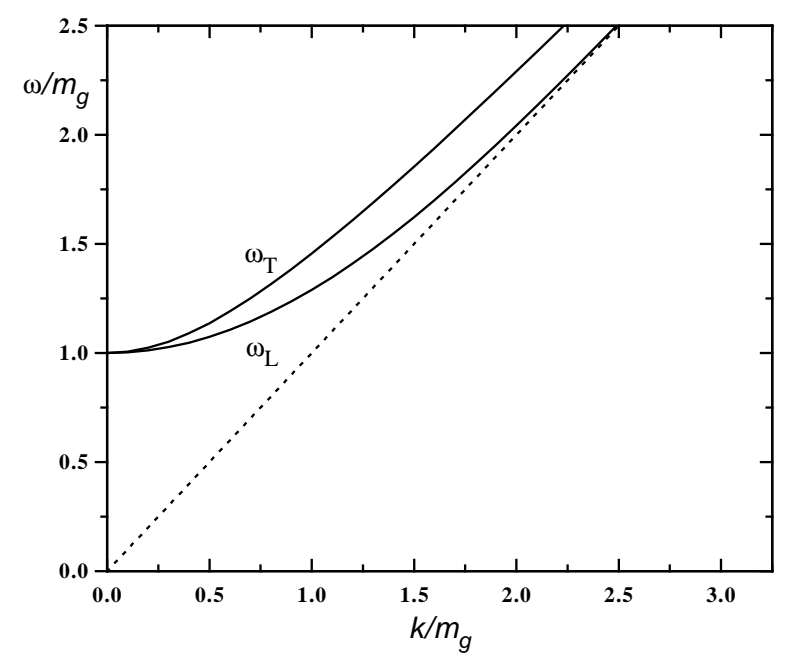

Fig. 4. Dispersion relation of longitudinal and transverse plasma waves in the QGP

in the QGP follow as shown in Fig.4. Both branches start at $k=0$ with the same energy $\omega_{L, T}(k=0)=m_{g}$, which can be identified with the plasma frequency. The longitudinal branch is known as plasmon. In the case of relativistic plasmas both branches are equally important, whereas in non-relativistic plasmas the transverse mode is usually neglected. Both modes approach the vacuum dispersion $\omega=k$ for large momenta. However, the spectral strength of the plasmon mode vanishes exponentially for large momenta, $k \gg g T$. Debye screening follows from the static limit $(\omega=0)$ of the longitudinal dielectric function describing the modification of the Coulomb potential to a Yukawa potential in the presence of a medium. The Debye screening length is given by $\lambda_{D}=1 /\left(\sqrt{3} m_{g}\right)$. The dielectric functions of (2) also contain Landau damping for $\omega^{2}<k^{2}$, for which the logarithms become imaginary. Although the plasma waves in the semiclassical approximation are located in the region $\omega^{2}>k^{2}$ (see Fig.4), where no Landau damping takes place, Landau damping of virtual gluons (plasma waves) in the QGP with $\omega^{2}<k^{2}$ plays an important role in higher order processes.

A further very interesting property of ultrarelativistic plasmas is the existence of collective fermion modes in analogy to the plasma modes, i.e. collective quark modes in the QGP. They can be derived within perturbation theory from the inmedium quark self-energy [10].

Finally, it is interesting to note that in the high temperature limit the non-abelian effects are negligible. Hence, apart from numerical factors, the above results hold also for an ultrarelativistic QED plasma, i.e. an electron-positron plasma in which the temperature is much higher than the electron rest mass. Such a situation is realized in Supernovae explosions, where temperatures up to $30 \mathrm{MeV}$ are possible. The dielectric functions of such a system are given by the above equation (2), in which one only has to replace the effective gluon mass $m_{g}$ by an effective photon mass $m_{\gamma}=e T / 3$.

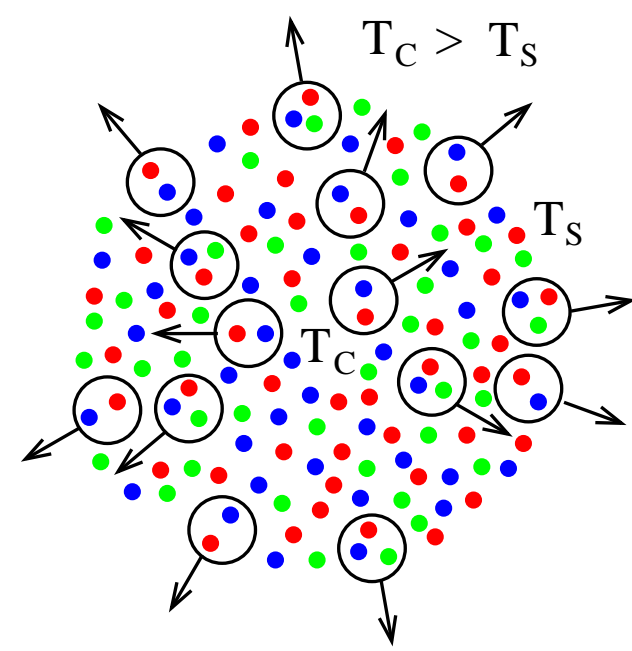

Fig. 5. Thermophoretic flow of hadrons in the mixed phase of the fireball

\section{Thermophoretic Flow in the Mixed Phase}

As a last point we want to consider a possible mixed phase of a QGP and hadrons. Such a mixed phase would occur if the phase transition from the QGP to the hadronic phase were of first order. During the expansion of the fireball in a heavy-ion collision or in the Early Universe, the mixed phase is reached, once the temperature has dropped to the critical. Then bubbles of hadrons will form (hadronization) and grow in the QGP until the entire phase consists of hadrons. QCD lattice simulations show that the order of the phase transition depends sensitively on the values of the up-, down-, and strange-quark masses. The latest calculations show that the QCD phase transition is either of first order or continuous, with a preference for the latter case [7].

Assuming a first order phase transition, the mixed phase can be considered as a complex plasma, in which extended and composite objects, the hadrons, are embedded in the QGP containing point-like partons, similar as dust grains in a lowtemperature plasma. The main difference lies in the fact that the dust particles are highly charged, whereas the hadrons are color-neutral objects. Now, we assume in addition to the mixed phase a temperature gradient in the fireball from the center to the surface and, for simplicity, a radial expansion of the fireball. Then, similar as in complex plasmas, a thermophoretic force will act on the hadrons, pushing them from the center to the surface as shown in Fig.5. The thermophoretic force in a complex plasma has been estimated from simple kinetic arguments and shown to be in excellent agreement with experimental results [11]. We have generalized the kinetic derivation of the thermophoretic force to the relativistic case and calculated the final velocity of the hadrons from this effect. Even for small temperature gradients of a few $\mathrm{MeV} / \mathrm{fm}$ and small life-times of the mixed phase $(1-2 \mathrm{fm} / \mathrm{c})$, we obtained large outward flow velocities of the order of $0.8 \mathrm{c}$ for pions and $0.4 \mathrm{c}$ for protons [12]. These values are larger than the typical flow velocities of $0.5 \mathrm{c}$, observed in relativistic heavyion collisions at SPS. We take this as an indication for the 
absence of a mixed phase in these experiments, either due to a continuous phase transition instead of first order or due to an absence of the QGP phase at all.

\section{REFERENCES}

[1] M. Gell-Mann, Phys. Lett. 8 (1964) 214

[2] H. Fritzsch, M. Gell-Mann, and H. Leutwyler, Phys. Lett. B 47 (1973) 365

[3] B. Müller, The Physics of the Quark-Gluon Plasma, Lecture Notes in Physics 225 (Springer, Berlin, 1985)

[4] Proc. of the 15th Int. Conf. on Ultra-Relativistic Nucleus-Nucleus Collisions "Quark Matter 2001", ed. T.J. Hallman, D.E. Kharzeev, J.T. Mitchell, and T. Ullrich, Nucl. Phys. A 698 (2002)

[5] M.Alford and S. Reddy, Phys. Rev. D 67 (2003) 074024; S.B. Rüster and D.H. Rischke, nucl-th/0309022

[6] U. Heinz and M. Jacob, nucl-th/0002042

[7] F. Karsch, AIP Conf. Proc. 631 (2003) 112

[8] M.H. Thoma, in:Quark-Gluon Plasma 2, ed.: R.C. Hwa (World Scientific, Singapore, 1995), p.51

[9] H.T. Elze and U. Heinz, Phys. Rep. 183 (1989) 81

[10] A. Peshier and M.H. Thoma, Phys. Rev. Lett. 84 (2000) 841

[11] H. Rothermel, T. Hagl, G.E. Morfill, M.H. Thoma, and H.M. Thomas, Phys. Rev. Lett. 89 (2002) 175001

[12] M.H. Thoma, Phys. Rev. Lett. 88 (2002) 202303 